\title{
Toxoplasma gondii Infection and Headache: A Matched Case-Control Study in a Public Hospital in Durango City, Mexico
}

\author{
Cosme Alvarado-Esquivele ${ }^{\mathrm{a}, \mathrm{e}}$, Yazmin del Rosario Rico-Almochantaf ${ }^{\mathrm{a}}$, Luis Francisco Sanchez-Anguiano ${ }^{\mathrm{b}}$, \\ Gerardo Quinones-Canales ${ }^{\mathrm{c}}$, Jesus Hernandez-Tinoco ${ }^{\mathrm{b}}$, Jorge Torres-Gonzalez ${ }^{\mathrm{c}}$, \\ Maria Felix Gonzalez-Silva ${ }^{\mathrm{d}}$, Eda Guadalupe Ramirez-Valles ${ }^{\mathrm{d}}$
}

\begin{abstract}
Background: Toxoplasma gondii (T. gondii) can disseminate to brain in infected hosts. Little is known about the magnitude of the association between this infection and headache. Therefore, we sought to determine the association of $T$. gondii seropositivity and headache in patients attending neurological consultations in a public hospital in Durango City, Mexico.

Methods: Through an age- and gender-matched case-control study, 105 patients suffering from headache and 105 subjects without headache were examined for anti-T. gondii $\operatorname{IgG}$ and IgM antibodies using commercially available enzyme-linked immunoassays. Seropositive cases were analyzed for detection of $T$. gondii DNA by polymerase chain reaction.

Results: Anti-T. gondii IgG antibodies were found in five (4.8\%) of the 105 cases and in seven (6.7\%) of the 105 controls (odds ratio (OR) $=0.70 ; 95 \%$ confidence interval $(\mathrm{CI}): 0.21-2.28 ; \mathrm{P}=0.76)$. The frequency of high ( $>150 \mathrm{IU} / \mathrm{mL}$ ) levels of anti- $T$. gondii $\mathrm{IgG}$ antibodies among anti- $T$. gondii $\mathrm{IgG}$ positive individuals was significantly $(\mathrm{P}=$ $0.01)$ higher in cases $(5 / 5)$ than in controls (1/7). Anti-T. gondii IgM antibodies were found in one $(20.0 \%)$ of the five IgG seropositive cases, and in three $(42.9 \%)$ of the seven IgG seropositive controls $(\mathrm{P}=0.60) . T$. gondii DNA was not detected in any of the five anti- $T$. gondii $\mathrm{IgG}$ positive cases. No association between $T$. gondii infection and specific headache types was found.
\end{abstract}

Manuscript submitted October 21, 2017, accepted October 31, 2017

${ }^{a}$ Biomedical Research Laboratory, Faculty of Medicine and Nutrition, Juarez University of Durango State, Avenida Universidad S/N, 34000 Durango, Mexico

bInstitute for Scientific Research "Dr. Roberto Rivera-Damm", Juarez University of Durango State, Avenida Universidad S/N, 34000 Durango, Mexico 'Instituto de Seguridad y Servicios Sociales para los Trabajadores del Estado, Predio Canoas S/N, 34000 Durango, Mexico

${ }^{\mathrm{d}}$ Faculty of Chemical Sciences, Juarez University of Durango State, Avenida Veterinaria S/N, 34120 Durango, Mexico

eCorresponding Author: Cosme Alvarado-Esquivel, Laboratorio de Investigacion Biomedica, Facultad de Medicina y Nutricion, Avenida Universidad S/N, 34000 Durango, Dgo, Mexico. Email: alvaradocosme@yahoo.com

doi: https://doi.org/10.14740/jocmr3236w
Conclusions: This is the first matched case-control study on the association between $T$. gondii infection and headache. Results suggest that high anti- $T$. gondii IgG antibody levels, but not $T$. gondii seropositivity, were associated with headache in the population studied.

Keywords: Toxoplasma gondii; Infection; Seroprevalence; Headache; Case-control study; Epidemiology; Mexico

\section{Introduction}

Toxoplasma gondii (T. gondii) is one of the most successful parasites in the world due to its ability to infect and persist in most warm-blooded animals [1]. About $30 \%$ of the human population worldwide is chronically infected with $T$. gondii [2]. Transmission of $T$. gondii may occur horizontally by ingestion of oocysts from the environment or ingestion of tissue cysts contained in meat from many different animals, vertically during pregnancy, by blood transfusion, and organ transplantation [3]. Infections in humans are commonly asymptomatic [2, 4]. When symptoms are present, they typically resemble a mononucleosis or flulike illness [5]. Infection with T. gondii may also lead to chorioretinitis [6]. Reactivation of a latent infection in immunodeficiency conditions can cause fatal toxoplasmic encephalitis [7]. When primary infection of the mother occurs during pregnancy, there is likelihood of a transplacental infection that can lead to congenital toxoplasmosis with affection of brain and retina [8].

T. gondii can persist in the central nervous system of a variety of hosts including humans [1]. The parasites persist as intraneuronal cysts that are controlled, but not eliminated by the immune system of the host [9]. Infection with T. gondii in brain in immunocompetent individuals may cause headache [10-12], confusion [10], and difficulty concentrating [12]. Headache of the migraine type has been linked to $T$. gondii infection [13]. In a cohort of 261 postnatal cases of acute toxoplasmosis in Brazil, headache was present in $10.7 \%$ of patients [14]. Very little is known about the frequency of $T$. gondii infection in patients suffering from headache. In a study of 108 cases of new daily persistent headaches in Dominican Republic, T. gondii infection was found in $3.7 \%$ of patients [15]. It is unclear how 
Table 1. Diagnoses of Headache and Frequency of T. gondii Infection in the Study Population

\begin{tabular}{|c|c|c|c|c|c|}
\hline \multirow{2}{*}{ ICD-10 code } & \multirow{2}{*}{ Diagnosis } & \multirow{2}{*}{ No. of patients } & \multicolumn{2}{|c|}{ Seropositivity to $T$. gondii } & \multirow{2}{*}{ P value } \\
\hline & & & No. & $\%$ & \\
\hline G43.0 & Migraine without aura & 16 & 2 & 12.5 & 0.74 \\
\hline G43.3 & Complicated migraine & 3 & 0 & 0 & \\
\hline G43.9 & Migraine, unspecified & 3 & 0 & 0 & \\
\hline G44.2 & Tension-type headache & 20 & 1 & 5 & \\
\hline G44.3 & Chronic post-traumatic headache & 3 & 0 & 0 & \\
\hline G44.8 & Other specified headache syndromes & 38 & 1 & 2.63 & \\
\hline
\end{tabular}

higher the frequency of $T$. gondii infection is in patients with headache as compared to subjects without headache. Therefore, we sought to determine the association between $T$. gondii infection and headache in patients attending at the Department of Neurology in a public hospital in the northern Mexican city of Durango.

\section{Materials and Methods}

\section{Study design and study populations}

We performed a case-control seroprevalence study of 105 patients suffering from headache (cases) and 105 people without headache from the general population (controls) in Durango City, Mexico. This study was performed from April 2016 to March 2017. Inclusion criteria for enrollment of the cases were: 1) patients suffering from headache attending in the Department of Neurology at the public Hospital "Dr. Santiago Ramon y Cajal" in Durango City; 2) aged 13 years and older; and 3) who voluntarily participate in the study. Only patients with diagnosis of headache made by two neurologists (GQC and JTG) were selected for inclusion in the study. Exclusion criterium was subjects suffering from a severe disease who were unable to decide themselves about their enrollment in the study. Gender, socioeconomic status, and occupation of patients were not restrictive criteria for inclusion in the study. In total, 82 females and 23 males suffering from headache were enrolled in the study. Cases were 13 - 86 years old $($ mean $=42.71 \pm 15.79)$. Diagnoses of headache in patients were based on the International Statistical Classification of Diseases and related Health Problems version 2016 (ICD-10) (http://apps.who.int/classifications/icd10/browse/2016/en). Table 1 shows the types of headache diagnosed in the studied patients. Concerning the control group, 105 subjects without headache were randomly selected from the general population in Durango City. All control subjects were matched with patients by age ( \pm 4 years) and gender. Controls were 15 - 86 years old (mean $=42.65 \pm 15.40)$, and included 82 females and 23 males. There was no difference in age between cases and controls $(\mathrm{P}=0.97)$.

\section{Detection of $T$. gondii IgG and IgM antibodies}

Sera of cases and controls were analyzed for anti-T. gondii IgG antibodies with the commercially available enzyme immunoassay kit "Toxoplasma IgG" (Diagnostic Automation Inc., Woodland Hills, CA, USA). This test allows qualitative and quantitative determinations of anti-T. gondii IgG antibodies. A cut-off of $\geq 8 \mathrm{IU} / \mathrm{mL}$ of specific anti- $T$. gondii IgG antibody was considered for seropositivity. IgG seropositive serum samples of cases and controls were further analyzed for anti- $T$. gondii IgM antibodies by the commercially available enzyme immunoassay "Toxoplasma IgM" kit (Diagnostic Automation Inc.). All IgG and IgM tests were performed following the instructions of the manufacturer. Positive and negative controls included in the kits were analyzed in each run. Criterium for diagnosis of $T$. gondii infection was based on results of the IgG test.

\section{DNA extraction and PCR of $T$. gondii}

Cases with anti-T. gondii IgG antibodies were further examined for detection of $T$. gondii DNA by nested-polymerase chain reaction (PCR). DNA extraction from whole blood was performed according to the instructions of a commercially available kit (QIAamp DNA Blood Mini kit; Qiagen, Germany). DNA amplification was performed with primers directed against the B1 gene of $T$. gondii and following the protocol described by Burg et al [16]. The PCR products were analyzed by using a $2 \%$ agarose gel electrophoresis stained with ethidium bromide. Visualization of the PCR products was performed with an ultraviolet transilluminator.

\section{Statistical analysis}

Statistical analyses were carried out using the Microsoft Excel 2010, SPSS version 15.0 (SPSS Inc. Chicago, IL, USA), and 
Epi Info version 7 (Centers for Disease Control and Prevention: http://wwwn.cdc.gov/epiinfo/) software. For calculation of the sample size, we used a 95\% two-sided confidence level, a power of $80 \%$, a 1:1 ratio of cases and controls, and a reference seroprevalence of $7.4 \%$ [17] as the expected frequency of exposure in controls. The result of the sample size calculation was 101 cases and 101 controls. The paired Student's $t$-test was used to compare age values among the groups. The association between seroprevalence and characteristics of cases was assessed by bivariate analysis. We calculated the odds ratios (ORs) and 95\% confidence intervals (CIs), and statistical significance was set at a $\mathrm{P}$ value less than 0.05 .

\section{Ethics aspects}

The Ethical Committee of the Institute of Security and Social Services for State Workers in Durango City, Mexico approved this project. The purpose and procedures of this case-control study were explained to all participants. A written informed consent was obtained from all subjects and from the next of kin of minor participants.

\section{Results}

Anti-T. gondii IgG antibodies were found in five (4.8\%) of the 105 cases and in seven $(6.7 \%)$ of the 105 controls. No difference in the seroprevalence of $T$. gondii infection between cases and controls was found $(\mathrm{OR}=0.70 ; 95 \% \mathrm{CI}: 0.21$ $2.28 ; \mathrm{P}=0.76)$. All five anti-T. gondii $\mathrm{IgG}$ positive cases had anti-T. gondii $\mathrm{IgG}$ antibody levels higher than $150 \mathrm{IU} / \mathrm{mL}$. In contrast, of the seven anti- $T$. gondii IgG positive controls, only one (14.3\%) had anti-T. gondii IgG antibody levels higher than $150 \mathrm{IU} / \mathrm{mL}$ (Table 2). The frequency of high $(>150$ $\mathrm{IU} / \mathrm{mL}$ ) levels of anti- $T$. gondii $\mathrm{IgG}$ antibodies among anti- $T$. gondii $\operatorname{IgG}$ positive individuals was significantly $(\mathrm{P}=0.01)$ higher in cases $(5 / 5)$ than in controls (1/7). Anti-T. gondii IgM antibodies were found in one $(20.0 \%)$ of the five IgG seropositive cases, and in three (42.9\%) of the seven IgG seropositive controls ( $\mathrm{P}=0.57$ by two-tailed Fisher exact test). T. gondii DNA was not detected in any of the five anti- $T$. gondii IgG positive cases.

Bivariate analysis of $\operatorname{IgG}$ seropositivity to $T$. gondii and ICD-10 codes did not show an association between $T$. gondii infection and specific types of headache (Table 1).

\section{Discussion}

The epidemiological impact of T. gondii infection on headache has been poorly studied. Some people infected with $T$. gondii suffer from headache [10-12]; however, it is unclear how many people suffering from headache has an infection with T. gondii. We are not aware of any age- and gender-matched case-control study on the association between $T$. gondii infection and headaches. Therefore, we decided to carry out this age- and gender-matched case-control study to determine the
Table 2. Anti-Toxoplasma IgG Levels in Cases and Controls

\begin{tabular}{|c|c|c|c|c|c|}
\hline \multirow{2}{*}{ Anti-Toxoplasma IgG levels } & \multicolumn{2}{|c|}{ Cases } & \multicolumn{2}{|c|}{ Controls } & \multirow{2}{*}{ P value } \\
\hline & No. & $\%$ & No. & $\%$ & \\
\hline$<100 \mathrm{IU} / \mathrm{mL}$ & 0 & 0 & 4 & 57.1 & 0.01 \\
\hline $100-150 \mathrm{IU} / \mathrm{mL}$ & 0 & 0 & 2 & 28.6 & \\
\hline$>150 \mathrm{IU} / \mathrm{mL}$ & 5 & 100 & 1 & 14.3 & \\
\hline
\end{tabular}

association of seropositivity to $T$. gondii and headache in patients attending at the Neurology Department in a public hospital in Durango City, Mexico. The seroprevalence of T. gondii infection in patients suffering from headache was low $(4.8 \%)$, and the seropositivity rate of anti-T. gondii $\operatorname{IgG}$ and $\operatorname{IgM}$ antibodies in patients suffering from headache and in control subjects was similar. The seroprevalence of $T$. gondii infection found in patients with headache is comparable to seroprevalences of $T$. gondii infection reported in the general population $(6.1 \%)$ [ [18], and healthy blood donors (7.4\%) [16] in the same Durango City. In contrast, the seroprevalence observed in patients suffering from headache is lower than seroprevalences of $T$. gondii infection reported in high risk groups in the same Durango City including waste pickers (21.1\%) [19], inmates $(21.1 \%)$ [20], and psychiatric patients (18.2\%) [21]. The low prevalence of $T$. gondii infection found in patients suffering from headache which is similar to or lower than those reported in controls and other population groups of the same city suggests that $T$. gondii infection did not contribute substantially for a higher risk of headache in our population studied. The low frequency of infection with $T$. gondii in patients with headache was unexpected since infection with $T$. gondii is considered a cause of headache in immunocompetent [1012], and immunocompromised patients [22]. Seroprevalence results suggest that factors other than $T$. gondii infection might contribute for headache in our population studied. No association between $T$. gondii infection and types of headache was found. However, the number of specific types of headache was small. Intriguingly, all five patients with headache and anti- $T$. gondii IgG antibodies had high ( $>150 \mathrm{IU} / \mathrm{mL}$ ) levels of anti- $T$. gondii IgG antibodies. This frequency was significantly higher than that found in control subjects. This finding might suggest a recent infection or a constant high antigenic stimulation. These results indicate an association between high anti-T. gondii IgG antibody concentration and headache. Very few studies have reported anti- $T$. gondii $\mathrm{IgG}$ concentrations in patients with headache. In a study in Poland, a high anti-T. gondii IgG concentration (mean: $189 \pm 85 \mathrm{IU} / \mathrm{mL}$ ) was found in patients with recurrent headache as the main symptom of acquired cerebral toxoplasmosis [11]. In a case report of a patient with systemic erythematosus lupus presenting with fever, headache, and mental change, high anti-T. gondii IgG titers in cerebrospinal fluid and in serum were found [23]. In the present study, no testing of anti-T. gondii IgM antibodies in subjects seronegative to anti- $T$. gondii $\mathrm{IgG}$ antibodies was performed. This was due to the low diagnostic value of an IgM positive test in subjects seronegative to IgG. A high number of false-positive IgM results have been reported using commercially available enzyme immunoassays for detection of anti-T. gondii IgM an- 
tibodies [24, 25].

The present study has some limitations. A small number of specific types of headache were studied. In addition, patients were enrolled in a single hospital, and most patients had a medium socioeconomic status. Further studies with larger number of patients with specific types of headache, enrolled in several hospitals or health care centers, and of several socioeconomic statuses to determine the association between $T$. gondii infection and headache should be conducted. Additional studies to determine the role of anti- $T$. gondii $\operatorname{IgG}$ concentration in headache are needed.

\section{Conclusions}

This is the first age- and gender-matched case-control study on the association between $T$. gondii infection and headache. Results suggest that high anti-T. gondii IgG antibody levels, but not $T$. gondii seropositivity, were associated with headache in the population studied. Because a small number of specific types of headache were studied, further studies to determine the association between $T$. gondii infection and types of headache are needed.

\section{Competing Interests}

The authors declare that they have no competing interests.

\section{Financial Support}

This study was financially supported by Secretary of Public Education, Mexico (Grant No. DSA/103.5/14/11311).

\section{References}

1. Mendez OA, Koshy AA. Toxoplasma gondii: Entry, association, and physiological influence on the central nervous system. PLoS Pathog. 2017;13(7):e1006351.

2. Schluter D, Daubener W, Schares G, Gross U, Pleyer U, Luder C. Animals are key to human toxoplasmosis. Int J Med Microbiol. 2014;304(7):917-929.

3. Tenter AM, Heckeroth AR, Weiss LM. Toxoplasma gondii: from animals to humans. Int J Parasitol. 2000;30(1213):1217-1258.

4. Feustel SM, Meissner M, Liesenfeld O. Toxoplasma gondii and the blood-brain barrier. Virulence. 2012;3(2):182192.

5. Beazley DM, Egerman RS. Toxoplasmosis. Semin Perinatol. 1998;22(4):332-338.

6. Aleixo AL, Curi AL, Benchimol EI, Amendoeira MR. Toxoplasmic retinochoroiditis: clinical characteristics and visual outcome in a prospective study. PLoS Negl Trop Dis. 2016;10(5):e0004685.

7. Saadatnia G, Golkar M. A review on human toxoplasmosis. Scand J Infect Dis. 2012;44(11):805-814.
8. Martin S. Congenital toxoplasmosis. Neonatal Netw. 2001;20(4):23-30.

9. Blanchard N, Dunay IR, Schluter D. Persistence of Toxoplasma gondii in the central nervous system: a fine-tuned balance between the parasite, the brain and the immune system. Parasite Immunol. 2015;37(3):150-158.

10. Akturk HK, Sotello D, Ameri A, Abuzaid AS, Rivas AM, Vashisht P. Toxoplasma infection in an immunocompetent host: possible risk of living with multiple cats. Cureus. 2017;9(3):e1103.

11. Prandota J. Recurrent headache as the main symptom of acquired cerebral toxoplasmosis in nonhuman immunodeficiency virus-infected subjects with no lymphadenopathy: the parasite may be responsible for the neurogenic inflammation postulated as a cause of different types of headaches. Am J Ther. 2007;14(1):63-105.

12. Wong WK, Upton A, Thomas MG. Neuropsychiatric symptoms are common in immunocompetent adult patients with Toxoplasma gondii acute lymphadenitis. Scand J Infect Dis. 2013;45(5):357-361.

13. Koseoglu E, Yazar S, Koc I. Is Toxoplasma gondii a causal agent in migraine? Am J Med Sci. 2009;338(2):120122.

14. Silva CS, Neves Ede S, Benchimol EI, Moraes DR. Postnatal acquired toxoplasmosis patients in an infectious diseases reference center. Braz J Infect Dis. 2008;12(5):438441.

15. Santoni JR, Santoni-Williams CJ. Headache and painful lymphadenopathy in extracranial or systemic infection: etiology of new daily persistent headaches. Intern Med. 1993;32(7):530-532.

16. Burg JL, Grover CM, Pouletty P, Boothroyd JC. Direct and sensitive detection of a pathogenic protozoan, Toxoplasma gondii, by polymerase chain reaction. J Clin Microbiol. 1989;27(8):1787-1792.

17. Alvarado-Esquivel C, Mercado-Suarez MF, RodriguezBriones A, Fallad-Torres L, Ayala-Ayala JO, NevarezPiedra LJ, Duran-Morales E, et al. Seroepidemiology of infection with Toxoplasma gondii in healthy blood donors of Durango, Mexico. BMC Infect Dis. 2007;7:75.

18. Alvarado-Esquivel C, Estrada-Martinez S, Pizarro-Villalobos H, Arce-Quinones M, Liesenfeld O, Dubey JP. Seroepidemiology of Toxoplasma gondii infection in general population in a northern Mexican city. J Parasitol. 2011;97(1):40-43.

19. Alvarado-Esquivel C, Liesenfeld O, Marquez-Conde JA, Cisneros-Camacho A, Estrada-Martinez S, Martinez-Garcia SA, Gonzalez-Herrera A, et al. Seroepidemiology of infection with Toxoplasma gondii in waste pickers and waste workers in Durango, Mexico. Zoonoses Public Health. 2008;55(6):306-312.

20. Alvarado-Esquivel C, Hernandez-Tinoco J, Sanchez-Anguiano LF, Ramos-Nevarez A, Cerrillo-Soto SM, SaenzSoto L, Liesenfeld O. High seroprevalence of Toxoplasma gondii infection in inmates: A case control study in Durango City, Mexico. Eur J Microbiol Immunol (Bp). 2014;4(1):76-82.

21. Alvarado-Esquivel C, Alanis-Quinones OP, Arreola-Valenzuela MA, Rodriguez-Briones A, Piedra-Nevarez LJ, 
Duran-Morales E, Estrada-Martinez S, et al. Seroepidemiology of Toxoplasma gondii infection in psychiatric inpatients in a northern Mexican city. BMC Infect Dis. 2006;6:178.

22. del Rio-Chiriboga C, Orzechowski-Rallo A, SanchezMejorada G. Toxoplasmosis of the central nervous system in patients with AIDS in Mexico. Arch Med Res. 1997;28(4):527-530.

23. Swaak AJ, Wouters JM, Portegies P, Sillevis Smitt PA. [Clinical thinking and decision making in clinical practice. A patient with systemic lupus erythemato- sus and behavioral changes]. Ned Tijdschr Geneeskd. 1998;142(10):513-517.

24. Liesenfeld O, Press C, Montoya JG, Gill R, Isaac-Renton JL, Hedman K, Remington JS. False-positive results in immunoglobulin $\mathrm{M}(\mathrm{IgM})$ toxoplasma antibody tests and importance of confirmatory testing: the Platelia Toxo IgM test. J Clin Microbiol. 1997;35(1):174-178.

25. Dhakal R, Gajurel K, Pomares C, Talucod J, Press CJ, Montoya JG. Significance of a positive toxoplasma immunoglobulin $\mathrm{M}$ test result in the United States. J Clin Microbiol. 2015;53(11):3601-3605. 